Performance characterization of a helicon double layer thruster using direct thrust measurements

This article has been downloaded from IOPscience. Please scroll down to see the full text article.

2011 J. Phys. D: Appl. Phys. 44235201

(http://iopscience.iop.org/0022-3727/44/23/235201)

View the table of contents for this issue, or go to the journal homepage for more

Download details:

IP Address: 131.227.8.198

The article was downloaded on 17/05/2011 at 09:52

Please note that terms and conditions apply. 


\title{
Performance characterization of a helicon double layer thruster using direct thrust measurements
}

\author{
Sabrina Pottinger ${ }^{1}$, Vaios Lappas ${ }^{1}$, Christine Charles $^{2}$ and Rod Boswell ${ }^{2}$ \\ ${ }^{1}$ Surrey Space Centre, University of Surrey, Guildford GU2 7XH, UK \\ ${ }^{2}$ Space Plasma, Power and Propulsion Group, Research School of Physics and Engineering, \\ The Australian National University, Canberra ACT 0200 Australia \\ Received 17 February 2011, in final form 28 March 2011 \\ Published 16 May 2011 \\ Online at stacks.iop.org/JPhysD/44/235201
}

\begin{abstract}
The performance of a helicon double layer thruster (HDLT) has been characterized using a pendulum type thrust stand and retarding field energy analyser. Data recorded for a fixed propellant flow rate of $16 \mathrm{sccm}$ of krypton and fixed magnetic field topology show that the thrust generated increases linearly with increasing radio frequency input power over a range $250-650 \mathrm{~W}$. Over the power range investigated thrust levels of approximately $1-2.8 \mathrm{mN}$ were achieved. A maximum effective specific impulse of $280 \mathrm{~s}$ was determined using the thrust data. Ion energy distribution functions indicate that increasing power corresponds to improved plasma generation processes as general trends show increasing plasma and beam currents with increasing power.
\end{abstract}

\section{Introduction}

The helicon double layer thruster (HDLT) is a relatively new electric propulsion concept that generates thrust by accelerating an RF generated plasma across a double layer that forms in the presence of a diverging magnetic field [1]. This technology offers advantages compared with conventional gridded ion engines and Hall effect thrusters in terms of the simplicity of the thruster design [2]. The lifetime of the HDLT is not limited by grid erosion mechanisms and the thruster produces a quasi neutral plume, therefore a hollow cathode or alternative beam neuralising device is made redundant. Proof of concept and thruster functionality has been demonstrated for a variety of propellants, magnetic field topologies and source tube dimensions [3-6].

A comprehensive evaluation of the HDLTs capabilities in terms of electric propulsion performance indicators such as thrust to power ratios and specific impulse $\left(I_{\mathrm{sp}}\right)$ has yet to be performed. This information is necessary for HDLT development and optimization for mission applications as well as providing data to aid in the understanding of the underlying physics of thruster operation. To date, direct thrust measurement data has not been published. Momentum flux measurements have been used to determine the thrust generated by a HDLT [7]. An instrument with a measurement range $0.02-0.5 \mathrm{mN}$ and resolution of $15 \mu \mathrm{N}$ measured a maximum thrust of $0.2 \mathrm{mN}$ for a thruster operating at a power of $100 \mathrm{~W}$, a mass flow rate of $25 \mathrm{sccm}$ of argon and a maximum magnetic field strength of $138 \mathrm{G}$, the source tube dimensions in this case were $150 \mathrm{~mm}$ diameter and $290 \mathrm{~mm}$ length. Indirect thrust measurements may provide an estimate of expected thrust levels but there are several issues associated with this measurement technique that can only be overcome with the use of direct measurement methods. The dimensions and placement of the target must be such that the entire cross section of the plasma plume impinges on the target. The insertion of a deflecting plate in the thruster plume results in plasma perturbations, the target will undergo charging and heating that will impact the properties of the plume environment. The impact and rebounding of ions and neutrals may also lead to erroneous results. These factors considered indirect thrust measurements often require verification by means of conventional direct thrust measurement, and discrepancies between the results determined using the two techniques have been demonstrated [8]. The current investigation aims to evaluate the performance of a HDLT for set operating parameters as a function of power and reports the first direct thrust measurements recorded for a HDLT.

\section{Experimental apparatus}

The evaluation of HDLT performance has been undertaken within a space simulation chamber capable of achieving 


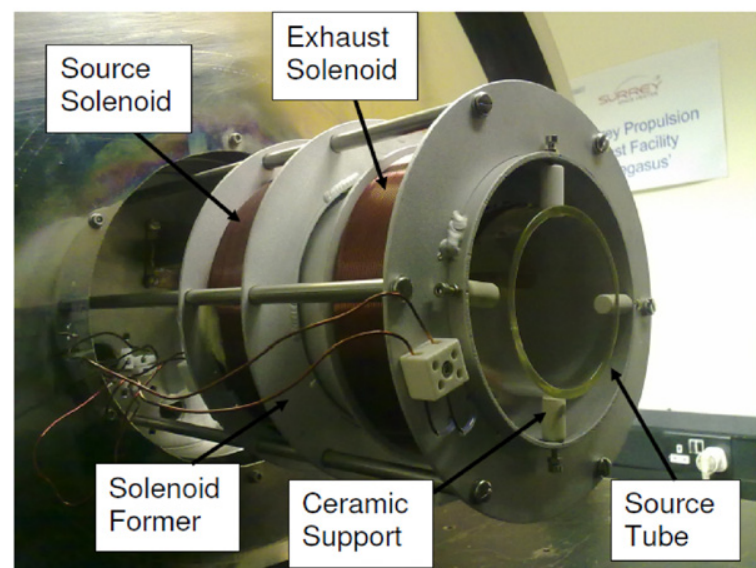

(a) The HDLT within the propulsion test facility

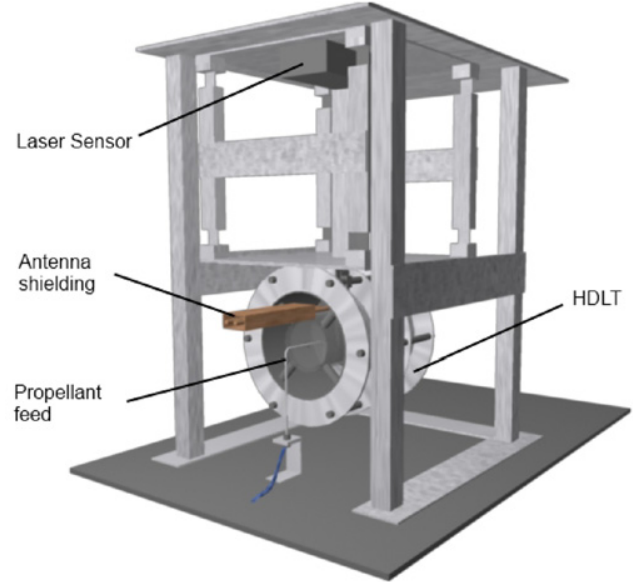

(b) CAD model of the HDLT mounted on the thrust stand. Courtesy of T. Harle

Figure 1. Experimental apparatus and the pendulum thrust stand.

(This figure is in colour only in the electronic version)

background pressures of the order of $10^{-7}$ mbar without load, the chamber has dimensions of $1.2 \mathrm{~m}$ length and $1.2 \mathrm{~m}$ diameter. The HDLT shown in figure 1(a) consists of a Pyrex source tube with an outer diameter of $80 \mathrm{~mm}$ and length $172 \mathrm{~mm}$. The source tube is surrounded by a source solenoid and an exhaust solenoid that terminates flush with the source tube exit. For all results presented the HDLT was operated using the exhaust solenoid alone to generate the necessary diverging magnetic field. An applied current of 3.5 A was supplied to the exhaust solenoid producing a peak magnetic field of approximately $100 \mathrm{G}$. A helicon discharge is generated by the deposition of radio frequency (RF) power into a copper antenna at a driving frequency of $13.56 \mathrm{MHz}$. The antenna type used in the current investigation is a double saddle antenna also known as a Boswell type antenna. The RF power is delivered via an impedance matching network incorporating two variable vacuum capacitors. The ratio of forward to reflected power is monitored using two independent methods: (1) a conventional standing wave meter and (2) the RF generator's in-built current-voltage sensor which accurately measures and displays load conditions. For all results presented the HDLT has been operated with krypton propellant at a flow rate of $16 \mathrm{sccm}\left(1 \mathrm{mg} \mathrm{s}^{-1}\right)$, which corresponds to a background pressure of $6.3 \times 10^{-4}$ mbar. Thrust measurements were recorded over an input power range $250-650 \mathrm{~W}$ in $50 \mathrm{~W}$ increments and RFEA measurements were taken for powers of 250-500 W.

\subsection{Thrust stand design}

An in house developed pendulum type thrust stand is used to characterize thruster performance, see figure $1(b)$. The thrust stand operates by comparing the deflection caused by the HDLT's applied thrust with the deflection caused by a calibrating force. The deflection of the thrust stand is measured with a laser optical displacement sensor with a resolution of $3 \mu \mathrm{m}$ at a sampling rate of $312.5 \mathrm{~Hz}$ to $2.5 \mathrm{kHz}$. The light from the semiconductor laser is deflected from a central support beam on the thrust stand and displacements are determined by the sensor unit via triangulation. A measuring rate of $312.5 \mathrm{~Hz}$ and 3 point averaging has been adopted for all testing.

A stepper motor and pulley apparatus has been incorporated into the thrust stand structure in order to allow in situ calibrations to take place during experimental trials. This apparatus functions by suspending a weight of a precisely known mass from a pulley that transfers this force directly to the thrust stand. The stepper motor can also be used to position the weight in a configuration where it applies zero force to the thrust stand. The response of the thrust stand to a static applied force over a range $1-10 \mathrm{mN}$ has been evaluated and shown that the response of the system is linear over the force range of interest. The standard uncertainty of the response of the thrust stand is approximately $\pm 3 \%$ of the measured value.

The calibration process also accounts for the impact of electrical harnessing. The system has been designed in order to minimize the number of mechanical connections to the thruster that could result in damping of the system. The only possible source of damping is the electrical connection to the HDLT solenoids, flexible wiring has been installed in order to mitigate this issue. The RF antenna and propellant feed line are mechanically isolated from the thruster. The fact that the propellant injector system is decoupled from the source means that the contribution of the expansion of neutral gas to the thrust is omitted from the measurement. This fact combined with the use of a non-optimized laboratory model thruster for this test campaign indicates that the thrust values presented represent the lower limit of HDLT performance.

\subsection{Ion energy distribution}

A retarding field energy analyser (REFA) has been used to record the ion energy distribution function of the HDLT plume. The probe consists of four electrically isolated grids (an earthed grid, repeller, discriminator, suppressor) and a collector plate, 


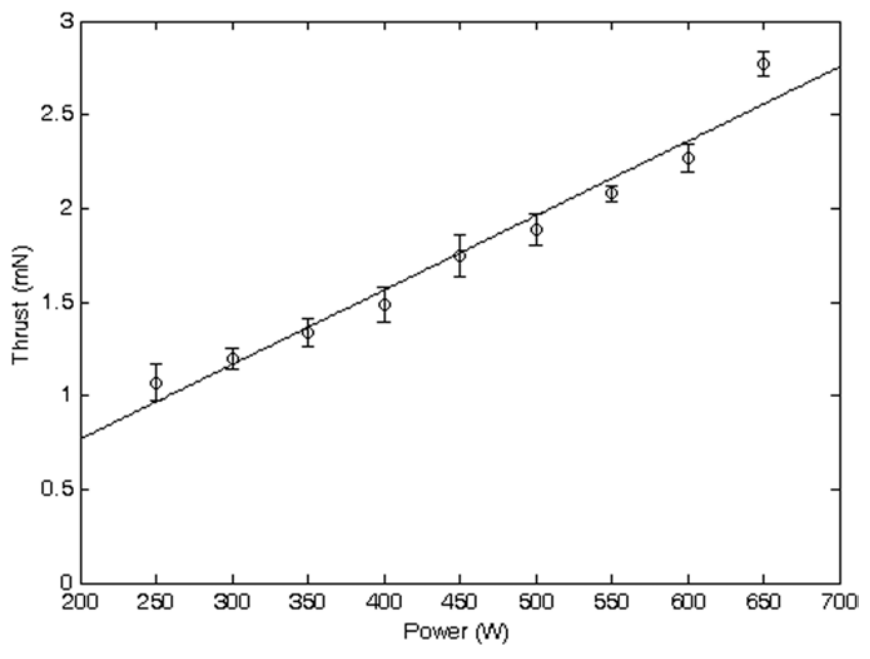

Figure 2. Thrust characterization for a krypton discharge with $\dot{m}=16 \mathrm{sccm}\left(1 \mathrm{mg} \mathrm{s}^{-1}\right)$ and applied solenoid current $3.5 \mathrm{~A}$.

which are sequentially stacked to form the probe head [9]. The probe orifice was placed on the thruster's central axis, all data was recorded at a single axial position approximately $5 \mathrm{~cm}$ downstream of the source tube exit. The positioning of the probe ensures that plasma perturbations caused by the presence of the RFEA do not interfere with the critical region in the vicinity of the source tube exit that is thought to be key in the formation of stable double layers. The influence of neutralion charge exchange collisions were also considered when selecting the probe position. Ion beams decay exponentially due to charge exchange collisions with neutrals [1]. As the mean free path for such collisions is of the order of $10 \mathrm{~cm}$ for the plasma conditions under investigation the probe was placed at half the mean free path to minimize these effects [7]. Ion energy distributions have been determined for input powers of $250-500 \mathrm{~W}$ and have been used to estimate the ion velocity of the HDLT plasma.

\section{Experimental results and discussion}

The thrust generated by a non-optimized laboratory prototype HDLT is presented in figure 2. For the test range investigated the data shows that thrust is a linear function of input RF power, each data point is the average result of 10 test runs in identical conditions. The main thrust generating mechanisms for the HDLT are the reaction forces produced as the ions accelerate across the double layer and then detach from the diverging magnetic field. The strength of the double layer is not highly dependent on RF power. The RFEA measurements presented in figure 3 correspond to a change in beam energy over the RF powers investigated of $\Delta E_{\text {beam }} \leqslant 1 \mathrm{eV}$. Theoretical studies verified by experiment have shown that the key parameter for double layer strength is background operating pressure which influences the ratio of ions to neutral species as well as the electron temperature [10]. The observed increase in thrust with increasing power may be attributed to increasing ion density at higher power and improved ion detachment. The beam current has been shown to increase linearly with power (see figure 4),

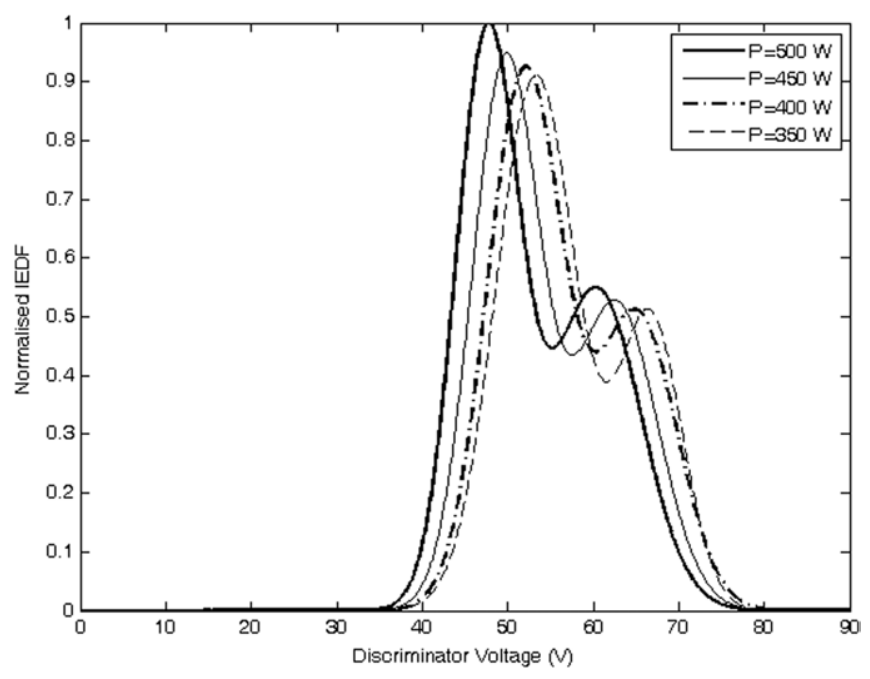

Figure 3. Normalised ion energy distribution functions for $\dot{m}=16 \mathrm{sccm} \mathrm{Kr}\left(1 \mathrm{mg} \mathrm{s}^{-1}\right)$ and applied solenoid current $3.5 \mathrm{~A}$.

as a consequence the number of ions contributing to the high ion energy population has increased. Many studies have been performed on the process of ion detachment, which is a crucial factor for the performance of electromagnetic thrusters. Gesto et al modelled ion trajectories in the plume of a HDLT and demonstrated that detachment occurs as the curvature of ion motion approaches zero. In this study electron dynamics were omitted, hence the electric field produced by ambipolar diffusion was neglected [11]. A two fluid plasma model was developed to investigate the optimization of ion detachment mechanisms, this study showed that the initial azimuthal velocity profile at the boundary of the exhaust aperture, i.e. the $r-z$ plane located at the source tube exit dictates the properties of an ions gyro motion and its capability to detach from the magnetic field [12]. Calculations of the gyroradius of electrons and ions in the plume of a HDLT using an experimentally determined electron temperature of $T_{e}=5.2 \pm 0.5 \mathrm{eV}$ show that it is of the order of the radius of the experiment [5]. Therefore, the plasma located downstream of the source tube exit which also corresponds to a region of weaker magnetic field strength is non-magnetized. These studies agree that the critical factor dictating ion detachment is the ion velocity on entering the plume region of the plasma which in turn dictates the rotational profile of ion motion. The application of RF power results in resonant high frequency fields being established that heat the plasma and increase the optimization rate, resulting in higher ion densities. Further work is required to relate the alignment of the RF electric and magnetic fields with respect to the velocity components of the ions, in this way the influence of applied RF power on ion detachment may be evaluated. It should also be noted that increasing the magnitude of RF input power may initiate plasma mode change. Mode change corresponds to jumps in plasma density as a consequence of improved power coupling, this phenomena may be manipulated to enhance thruster performance [13].

Figure 3 shows the ion energy distribution functions for the HDLT plasma for various input power levels. The data is normalized to the peak current produced for an RF input 


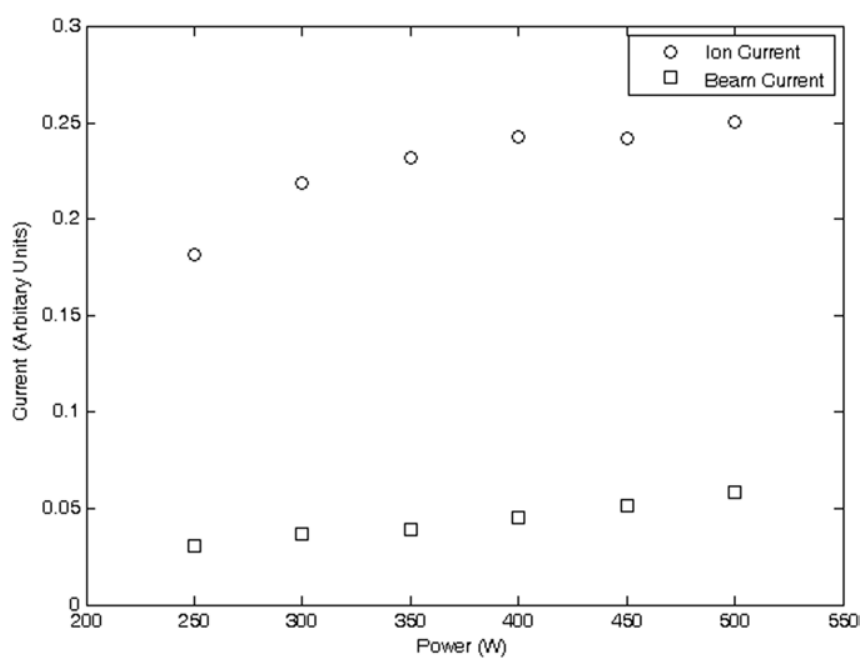

Figure 4. The HDLT ion current (circles) and beam current (squares) recorded $5 \mathrm{~cm}$ downstream of the source tube exit.

power of $500 \mathrm{~W}$. All distributions show two peaks which is characteristic of the presence of a high energy ion population (the ion beam) superimposed on the bulk plasma population. It can be seen that increasing power from 350 to $500 \mathrm{~W}$ results in a shift in plasma and beam potential to lower potentials. Increases in the collected ion current and beam current are observed with increasing power. The ratio of the number densities of the high energy plasma population to bulk plasma population increases with increasing power, which is confirmed by the data presented in figure 4 . The ion current shown in figure 4 corresponds to the peak current collected by the RFEA at the plasma potential and the beam current is the current corresponding to the beam potential. The collected ion and beam currents do not display a strong dependence on the input power. The ion current plateaus for powers greater than $350 \mathrm{~W}$ and the beam current displays a linear relationship with power. This indicates that at higher powers a greater proportion of the plasma contributes to the higher energy population than observed at lower power levels.

Assuming the HDLT plasma consists of a monoenergetic beam with zero beam divergence the ion velocity $v_{\text {ion }}$ may be determined applying the conservation of energy shown by equation (1):

$$
\frac{1}{2} m v_{\text {ion }}^{2}=e\left(V_{\mathrm{b}}-V_{\mathrm{p}}\right),
$$

Where $m$ is the ion mass, $V_{\mathrm{p}}$ is the plasma potential, $V_{\mathrm{b}}$ is the beam potential and the elementary charge, $e=1.602 \times$ $10^{-19} \mathrm{C}$. The effective exhaust velocity $v_{\text {exh }}$ is determined for a measured thrust $T$ at a mass flow rate $\dot{m}$ where $I_{\mathrm{sp}}$ is the specific impulse and $g$ is the acceleration due to gravity at the Earth's surface:

$$
I_{\mathrm{sp}}=\frac{T}{g \dot{m}}=\frac{v_{\mathrm{exh}}}{g} .
$$

The values of ion velocity presented in figure 5 determined using measured beam energies in conjunction with equation (1) show good agreement with the velocity determined in previous investigations where $v_{\text {ion }} \sim 6 \mathrm{~km} \mathrm{~s}^{-1}$ was found for a HDLT operating with xenon propellant [14]. Using equation (2)

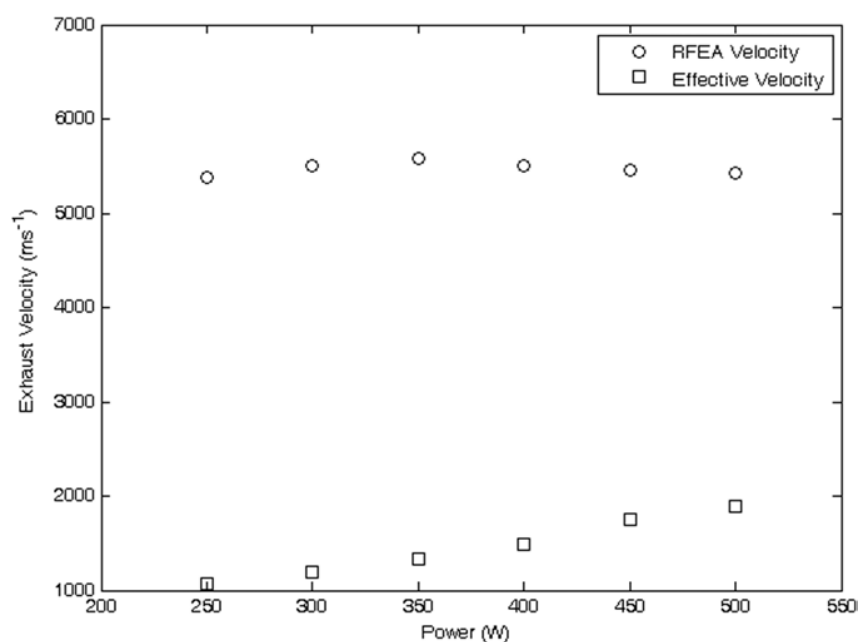

Figure 5. The exhaust velocity determined from the beam energy (circles) and the effective velocity (squares).

to determine the expected thrust for ion velocities shown in figure 5 results in $T \sim 5 \mathrm{mN}$. A maximum thrust of $2.8 \mathrm{mN}$ was measured using the apparatus described in section 2.1, this corresponds to an effective specific impulse of $280 \mathrm{~s}$ which is determined using equation (2). The difference between the ion velocity determined using the RFEA and the effective exhaust velocity calculated via direct measurement may be attributed to the assumptions made in the calculation of $v_{\text {ion }}$ with the use of equation (1). By assuming the beam is fully ionized the impact of neutral species which leave the source channel with a thermal velocity is neglected, the beam is also assumed to have zero divergence. These factors result in an over estimate of the exhaust velocity and calculated thrust. From equation (2) we may define the thrust as $T=\dot{m} v_{\text {exh }}$, taking the ratios of the exhaust velocities determined using the RFEA and direct measurement provides the ratio of the ion flow rate and the mass flow rate which describes the mass utilization of the HDLT. For the test conditions under investigation a minimum mass utilization of $20 \%$ is achieved for a RF input power of $250 \mathrm{~W}$ and $35 \%$ is achieved at $500 \mathrm{~W}$. This is a key result which confirms that in order to improve the performance of the HDLT the ion fraction must be increased. The thrust and specific impulse of conventional electric thrusters with proven flight heritage is significantly greater than the levels demonstrated by the non-optimized laboratory model HDLT. Electron bombardment ion thrusters such as the T6 by Qinetic, NSTAR and XIPS - 25 thrusters have demonstrated a specific impulse of $4120 \mathrm{~s}, 3100 \mathrm{~s}$ and $3800 \mathrm{~s}$, respectively $[15,16]$. These thrusters operate at powers in excess of $4 \mathrm{~kW}$ and achieve thrust to power ratios of approximately $0.03 \mathrm{mN} \mathrm{W}^{-1}$ compared with $0.004 \mathrm{mN} \mathrm{W}^{-1}$ produced by the HDLT in this investigation. Hall effect thrusters have shown a throttling capability of $75-250 \mathrm{mN}$ corresponding to a nominal specific impulse of $1500 \mathrm{~s}$ over a power range $1.5-6 \mathrm{~kW}$ [17]. Gridded radio frequency ion thrusters such as the RIT series produce thrust ranging from 10 to $250 \mathrm{mN}$ with increasing discharge chamber diameter [18]. The RIT 10 which operates at input powers comparable to the HDLT is a $10 \mathrm{mN}$ thruster with a specific impulse of $3300 \mathrm{~s}$. It should be noted that one of the 
key advantages of the HDLT is the capability to accelerate ions without the use of a system of grids. This process eliminates a wear mechanism but also limits the energy of the accelerated ions to the potential of the double layer. Although this self imposed limit prevents the HDLT from achieving specific impulse comparable to that achieved by established technologies this limit prevents damage to the spacecraft due to sputtering by high energy ions which is advantageous from a systems engineering perspective. The HDLT remains at an early stage of development and this initial characterization highlights areas for future work and performance enhancement.

\section{Conclusions}

This investigation has demonstrated the linear relationship between thrust and applied RF power for a HDLT using direct thrust measurements. The observed increase in thrust is supported by probe measurements which indicate the number of beam ions comprising the high energy population of the plasma increases with increasing power. These results provide confirmation that ion detachment occurs and is consistent with the results reported in literature. Double layer strength has not varied greatly as a function of power, therefore maximizing propellent utilization efficiency and ion detachment are critical factors for enhancing thruster performance. For the test conditions investigated a propulsive thrust efficiency $\left(\eta_{\mathrm{T}}=T^{2} / 2 \dot{m} P\right)$ of $\eta_{\mathrm{T}} \leqslant 1 \%$ is attained. The low thrust efficiency reiterates the fact that ion fraction must be increased and the thruster requires further optimization in order to provide a viable alternative to conventional electric propulsion technologies. Based on the indication that HDLTs possess the capability to operate over extended lifetimes future mission applications for this technology may be interplanetary scientific missions or in the long term cargo transfer missions where rapid transfer times are not imposed. On orbit applications such as orbit maintenance and reorbit at end of life are also feasible for missions requiring on orbit operations in excess of 15 years. In order to provide a competitive propulsion option thruster performance optimization must take place accompanied by an increased technology readiness level.

Further work is required to confirm the physics of thrust generating mechanisms, in particular combining the processes associated with double layer acceleration and ion detachment. The relative contribution of each thrust generation mechanism to the net thrust should also be evaluated. Experimental characterization of HDLTs for various operating parameters is necessary to identify high performance regimes and provide reliable data for the validation of theoretical studies. This data is crucial for the optimization of HDLT designs and developing the fundamental physics of thruster operation.

\section{Acknowledgment}

This research has been funded through the University of Surrey's strategic partnership agreement with EADS Astrium.

\section{References}

[1] Charles C 2009 Plasmas for spacecraft propulsion J. Phys. D: Appl. Phys. 42163001

[2] Martinez-Sanchez M and Pollard J E 1998 Spacecraft electric propulsion-an overview J. Propulsion Power 14 688-99

[3] Charles C, Boswell R W, Laine R and MacLellan P 2008 An experimental investigation of alternative propellants for the helicon double layer thruster J. Phys. D: Appl. Phys. 41175213

[4] Charles C and Boswell R W 2008 Effect of exhaust magnetic field in a helicon double-layer thruster operating in xenon IEEE Trans. Plasma Sci. 36 2141-6

[5] West M D, Charles C and Boswell R W 2008 Testing a helicon double layer thruster immersed in a space-simulation chamber J. Propulsion Power 24 134-41

[6] Pottinger S J, Harle T, Lappas V J, Cox W, Lafleur T, Alexander P, Charles C, Boswell R W, Perren M and Laine R 2010 Helicon double layer thruster development for low power operation Proc. Space Propulsion 2010 (San Sebastian, Spain) pp 1-10

[7] West M D, Charles C and Boswell R W 2009 A high sensitivity momentum flux measuring instrument for plasma thruster exhausts and diffusive plasmas Rev. Sci. Instrum. 80053509

[8] Longmier B W, Chavers G and Bering III E A 2009 Validating a plasma momentum flux sensor to an inverted pendulum thrust stand J. Propulsion Power 25 746-52

[9] Rudakov D L, Shats M G, Boswell R W, Charles C and Howard J 1999 Overview of probe diagnostics on the H-1 heliac Rev. Sci. Instrum. 70 476-9

[10] Charles C 2007 A review of recent laboratory double layer experiments Plasma Sources Sci. Technol. 16 R1-R25

[11] Gesto F N, Blackwell B D, Charles C and Boswell R W 2006 Ion detachment in the helicon double-layer thruster exhaust beam J. Propulsion Power 22 24-30

[12] Schmit P F and Fisch N J 2009 Magnetic detachment and plume control in escaping magnetized plasma J. Plasma Phys. 75 359-71

[13] Franck C M, Grulke O and Klinger T 2003 Mode transitions in helicon discharges Phys. Plasmas $10323-5$

[14] Charles C, Boswell R W and Lieberman M A 2006 Xenon ion beam characterization in a helicon double layer thruster Appl. Phys. Lett. 89261503

[15] Snyder J S, Goebel D M, Hofer R R, Polk J E, Wallace N C and Simpson H 2010 Performance evaluation of the T6 ion engine 46th AIAA/ASME/SAE/ASEE Joint Propulsion Conf. and Exhibit (Nashville, TN) AIAA 2010-7114:1-13

[16] Wilbur P J, Rawlin V K and Beattie J R 1998 Ion thruster development trends and status in the United States $J$. Propulsion Power 4 708-14

[17] Walker M L R and Gallimore A D 2007 Performance characteristics of a cluster of 5-kW laboratory Hall thrusters J. Propulsion Power 23 35-43

[18] Groh K H and Loeb H W 1994 State of the art of radio-frequency ion sources for space propulsion Rev. Sci. Instrum. 65 1741-4 